\title{
CONSTRUCTIONS OF THE MAXIMAL STRONGLY CHARACTER INVARIANT SEGAL ALGEBRAS AND THEIR APPLICATIONS
}

\author{
CHANG-PAO CHEN
}

(Received 26 March 1982)

Communicated by W. Moran

\begin{abstract}
Let $G$ denote any locally compact abelian group with the dual group $\Gamma$. We construct a new kind of subalgebra $L^{\prime}(G) \otimes_{\Gamma} S$ of $L^{1}(G)$ from given Banach ideals $S$ in $L^{\prime}(G)$. We show that $L^{1}(G) \otimes_{\Gamma} S$ is the largest among all strongly character invariant homogeneous Banach algebras in $S$. When $S$ contains a strongly character invariant Segal algebra on $G$, it is shown that $L^{\prime}(G) \otimes_{\Gamma} S$ is also the largest among all strongly character invariant Segal algebras in $S$. We give applications to characterizations of two kinds of subalgebras of $L^{1}(G)$-strongly character invariant Segal algebras on $G$ and Banach ideals in $L^{\prime}(G)$ which contain a strongly character invariant Segal algebra on $G$.
\end{abstract}

1980 Mathematics subject classification (Amer. Math. Soc.): primary 22 B 10, 43 A 20; secondary 43 A $15,46 \mathrm{~J} 15$.

Keywords and phrases. Banach ideal, homogeneous Banach algebra, Segal algebra, strongly character invariant, locally compact abelian group.

\section{Notations and definitions}

Throughout this article, $T$ denotes the circle group. $R$ denotes the additive group of real numbers. $G$ denotes any locally compact abelian group with the dual group $\Gamma$. $P\left(L^{1}(G)\right)$ denotes the space of all $f$ in $L^{1}(G)$ whose Fourier transforms $\hat{f}$ have compact support.

For the convenience of the readers, we recall some definitions: An ideal $S$ in $L^{1}(G)$ is called a normed ideal in $L^{\prime}(G)$ if $S$ is also a normed linear space under some norm \|\|$_{S}$ such that $\|f * g\|_{S} \leqslant\|f\|_{1}\|g\|_{S}$ for all $f \in L^{1}(G)$ and $g \in S$.

(c) Copyright Australian Mathematical Society 1983 
This definition is weaker than that of $\mathrm{J}$. Cigler [2]. In addition, if $\left(S,\|\|_{S}\right)$ is also a Banach space, then $S$ is called a Banach ideal in $L^{1}(G)$. A subalgebra $S$ of $L^{1}(G)$ is called a semi-homogeneous Banach algebra on $G$ if $S$ is a Banach algebra under some norm \|\|$_{S} \geqslant\|\|_{1}$ and satisfies the property:

$(H-1)$ If $f \in S$ and $x \in G$, then $L_{x} f \in S$ and $\left\|L_{x} f\right\|_{S}=\|f\|_{S}$ (where $L_{x} f(y)$ $=f(y-x))$.

If $S$ satisfies the additional property:

$(H-2)$ For every $f \in S$, the map $x \rightarrow L_{x} f$ is continuous from $G$ into $\left(S,\|\|_{S}\right)$, then $S$ is called a homogeneous Banach algebra on $G$. The definition is equivalent to that of a homogeneous Banach space in Katznelson [6]. The proof can be found in [13, Theorem 3.2]. A semi-homogeneous Banach algebra $S$ on $G$ is called (strongly) character invariant if $\gamma \in \Gamma, f \in S$ imply $\gamma f \in S$ (and $\|\gamma f\|_{S}=\|f\|_{S}$ ), where $\gamma f(x)=(x, \gamma) f(x)$. In [13], H. C. Wang uses the word "character" instead of "strongly character invariant". A dense homogeneous Banach algebra in $L^{\prime}(G)$ is called a Segal algebra on $G$. For fundamental results on Segal algebras, see Reiter ([8,9]) and Wang [13].

\section{Construction of the maximal strongly character invariant homogeneous Banach algebras}

Suppose that $\left(A,\|\|_{A}\right)$ and $\left(B,\|\|_{B}\right)$ are two normed linear spaces in $L^{1}(G)$ with \|\|$_{A} \geqslant\|\|_{1}$ and \|\|$_{B} \geqslant\|\|_{1}$. We introduce a new kind of linear subspaces of $L^{\mathrm{l}}(G)$ as follows: The set $A \otimes^{\mathrm{\Gamma}} B$ consists of all those elements $f \in L^{\mathrm{l}}(G)$ such that

$$
f=\sum_{n} g_{n} * h_{n}
$$

subject to the conditions:

(ii) $\gamma g_{n} \in A \cap P\left(L^{1}(G)\right), \quad \gamma h_{n} \in B \cap P\left(L^{1}(G)\right), \quad \forall n \geqslant 1, \gamma \in \Gamma$;

and

$$
\sup _{\gamma \in \Gamma} \sum_{n}\left\|\gamma g_{n}\right\|_{A}\left\|\gamma h_{n}\right\|_{B}<\infty .
$$

Clearly, (iii) implies that the series (i) converges in $L^{1}(G)$ and

$$
\|f\|_{1} \leqslant \text { the infimum of all possible values in (iii). }
$$

Denote the infimum by $\|f\|_{\Gamma}$. The above inequality means that

$$
\|f\|_{1} \leqslant\|f\|_{\Gamma} \text {. }
$$


We remark here that $A \otimes{ }^{\Gamma} B$ may be zero. For example, take $A=L^{1}(T)$ and $B=C^{1}(T)=$ the space of all continuously differentiable functions on $T$. It follows from the above definitions that $A \otimes^{\Gamma} B=B \otimes \Gamma A$ and $\left(A \otimes{ }^{\Gamma} B,\|\|_{\Gamma}\right)$ is a normed linear space in $L^{\prime}(G)$. Moreover,

Proposition 1. Let $A$ be a normed ideal in $L^{\prime}(G)$. If $A$ or $B$ satisfies the $(H-1)$ property, then $A \otimes{ }^{\Gamma} B$ is not only a strongly character invariant semi-homogeneous Banach algebra on $G$ but also a normed ideal in $L^{1}(G)$.

Proof. Let $\left(f_{m}\right)$ be a sequence in $A \otimes^{\Gamma} B$ with $\Sigma_{m}\left\|f_{m}\right\|_{\Gamma}<\infty$. For each $m$ there exists two sequences $\left(g_{n}^{m}\right) \subseteq A \cap P\left(L^{1}(G)\right)$ and $\left(h_{n}^{m}\right) \subseteq B \cap P\left(L^{1}(G)\right)$ such that

$$
f_{m}=\sum_{n} g_{n}^{m} * h_{n}^{m}
$$

and

$$
\sup _{\gamma \in \Gamma} \sum_{n}\left\|\gamma g_{n}^{m}\right\|_{A}\left\|\gamma h_{n}^{m}\right\|_{B} \leqslant\left\|f_{m}\right\|_{\Gamma}+2^{-m} .
$$

This implies that

$$
\begin{aligned}
\sum_{m} \sum_{n}\left\|g_{n}^{m}\right\|_{1}\left\|h_{n}^{m}\right\|_{1} & \leqslant \sup _{\gamma \in \Gamma} \sum_{m} \sum_{n}\left\|\gamma g_{n}^{m}\right\|_{A}\left\|\gamma h_{n}^{m}\right\|_{B} \\
& \leqslant \sum_{m}\left(\sup _{\gamma \in \Gamma} \sum_{n}\left\|\gamma g_{n}^{m}\right\|_{A}\left\|\gamma h_{n}^{m}\right\|_{B}\right) \\
& \leqslant \sum_{m}\left(\left\|f_{m}\right\|_{\Gamma}+2^{-m}\right)<\infty .
\end{aligned}
$$

Let $f=\Sigma_{m} \Sigma_{n} g_{n}^{m} * h_{n}^{m}$ in $\left(L^{1}(G),\|\|_{1}\right)$. From the above inequalities we find that

$$
f-\sum_{1 \leqslant k \leqslant m} f_{k}=\sum_{k>m} \sum_{n} g_{n}^{k} * h_{n}^{k} \quad \text { in }\left(L^{1}(G),\|\|_{1}\right)
$$

and

$$
\begin{aligned}
\left\|f-\sum_{1 \leqslant k \leqslant m} f_{k}\right\|_{\Gamma} & \leqslant \sup _{\gamma \in \Gamma} \sum_{k>m} \sum_{n}\left\|\gamma g_{n}^{k}\right\|_{A}\left\|\gamma h_{n}^{k}\right\|_{B} \\
& \leqslant \sum_{k>m}\left(\sup _{\gamma \in \Gamma} \sum_{n}\left\|\gamma g_{n}^{k}\right\|_{A}\left\|\gamma h_{n}^{k}\right\|_{B}\right) \\
& \leqslant \sum_{k>m}\left(\left\|f_{k}\right\|_{\Gamma}+2^{-k}\right) \\
& \rightarrow 0 \quad \text { as } m \rightarrow \infty
\end{aligned}
$$


which shows that the series $\Sigma_{m} f_{m}$ converges to $f$ in $\left(A \otimes \Gamma B,\|\|_{\Gamma}\right)$, and so $\left(A \otimes{ }^{\Gamma} B,\|\|_{\Gamma}\right)$ is complete. The remainder of the proof is based on the identities: $\gamma(f * g)=(\gamma f) *(\gamma g)$ and $\gamma\left(L_{x} f\right)=(x, \gamma) L_{x}(\gamma f)$. It is so straightforward as to be omitted.

Proposition 2. Suppose that $A$ and $B$ satisfy the hypotheses of Proposition 1. Let $A \otimes_{\Gamma} B$ denote the space of all $f \in A \otimes \otimes^{\Gamma} B$ such that $x \rightarrow L_{x} f$ is continuous from $G$ into $\left(A \otimes \Gamma B,\|\|_{\Gamma}\right)$; then $\left(A \otimes_{\Gamma} B,\|\|_{\Gamma}\right)$ is a strongly character invariant homogeneous Banach algebra on $G$.

Proof. In view of [13, Theorem 2.6], it suffices to show that $\gamma \in \Gamma, f \in A \otimes_{\Gamma} B$ imply $\gamma f \in A \otimes_{\Gamma} B$. We have

$$
\begin{aligned}
\left\|L_{x}(\gamma f)-L_{y}(\gamma f)\right\|_{\Gamma}= & \left\|(-x, \gamma) \gamma L_{x} f-(-y, \gamma) \gamma L_{y} f\right\|_{\Gamma} \\
\leqslant & |(-x, \gamma)-(-y, \gamma)|\left\|\gamma L_{x} f\right\|_{\Gamma} \\
& +|(-y, \gamma)|\left\|\gamma\left(L_{x} f-L_{y} f\right)\right\|_{\Gamma} \\
= & |(-x, \gamma)-(-y, \gamma)|\|f\|_{\Gamma}+\left\|L_{x} f-L_{y} f\right\|_{\Gamma} .
\end{aligned}
$$

Since $\gamma$ is continuous on $G$ and $f \in A \otimes_{\Gamma} B$, it follows that $x \rightarrow L_{x}(\gamma f)$ is continuous from $G$ into $\left(A \otimes^{\Gamma} B,\|\|_{\Gamma}\right)$ and so $\gamma f \in A \otimes_{\Gamma} B$. This completes the proof.

Proposition 3. If $A$ and $B$ are two Banach ideals in $L^{1}(G)$, then $A \otimes_{\Gamma} B \subseteq$ $A \otimes \Gamma^{\Gamma} B \subseteq A \cap B$ and \|\|$_{\Gamma} \geqslant \max \left(\|\|_{A},\|\|_{B}\right)$.

REMARK. This proposition does not hold in case of normed ideals, that is, it is necessary that $\left(A,\|\|_{A}\right)$ and $\left(B,\|\|_{B}\right)$ be complete. For example, consider ( $A$, \|\|$\left._{A}\right)=\left(L^{1}(G),\|\|_{1}\right)$ and $\left(B,\|\|_{B}\right)=\left(P\left(L^{1}(G)\right),\|\|_{1}\right)$. Here $\left(B,\|\|_{B}\right)$ is not complete. It follows easily that $f \in A \otimes^{\Gamma} B$ and $\|f\|_{\Gamma}=\|f\|_{1}$ for all $f \in$ $P\left(L^{1}(G)\right)$, which implies $A \otimes_{\Gamma} B=A \otimes{ }^{\Gamma} B=L^{1}(G) \not \subset A \cap B$.

Proof of Proposition 3. For the sake of symmetry, it suffices to show that $A \otimes{ }^{\Gamma} B \subseteq A$ and \|\|$_{A} \leqslant\|\|_{\Gamma}$. Let $f \in A \otimes{ }^{\Gamma} B$. For any $\varepsilon>0$, there exist $\left(g_{n}\right) \subseteq A \cap P\left(L^{1}(G)\right)$ and $\left(h_{n}\right) \subseteq B \cap P\left(L^{1}(G)\right)$ such that

$$
f=\sum_{n} g_{n} * h_{n}
$$

and

$$
\sup _{\gamma \in \Gamma} \sum_{n}\left\|\gamma g_{n}\right\|_{A}\left\|\gamma h_{n}\right\|_{B}<\|f\|_{\Gamma}+\varepsilon .
$$


We have

$$
\begin{aligned}
\sum_{n}\left\|g_{n} * h_{n}\right\|_{A} & \leqslant \sum_{n}\left\|g_{n}\right\|_{A}\left\|h_{n}\right\|_{1} \leqslant \sum_{n}\left\|g_{n}\right\|_{A}\left\|h_{n}\right\|_{B} \\
& \leqslant \sup _{\gamma \in \Gamma} \sum_{n}\left\|\gamma g_{n}\right\|_{A}\left\|\gamma h_{n}\right\|_{B} \\
& >\|f\|_{\Gamma}+\varepsilon<\infty .
\end{aligned}
$$

This implies that there exists $\phi \in A$ such that $\phi=\sum_{n} g_{n} * h_{n}$ in $\left(A,\|\|_{A}\right)$ and consequently $\phi=\Sigma_{n} g_{n} * h_{n}$ in $\left(L^{1}(G)\right.$, \|\|$\left._{1}\right)$. Since $f=\Sigma_{n} g_{n} * h_{n}$ in $\left(L^{\prime}(G)\right.$, \|\|$_{1}$ ), it follows that $\phi=f$. Therefore $A \otimes^{\Gamma} B \subseteq A$. On the other hand,

$$
\|f\|_{A}=\|\phi\|_{A} \leqslant \sum_{n}\left\|g_{n} * h_{n}\right\|_{A}<\|f\|_{\Gamma}+\varepsilon .
$$

It follows that $\|f\|_{A} \leqslant\|f\|_{\Gamma}$. This completes the proof.

TheOREM 4. Let $S$ be a Banach ideal in $L^{\prime}(G)$, then $L^{1}(G) \otimes_{\Gamma} S$ is the largest among all strongly character invariant homogeneous Banach algebras in $S$.

REMARK. In general, $L^{1}(G) \otimes_{\Gamma} S$ is smaller than the maximal homogeneous Banach space $S_{c}$ in $S$. (See [6] and [13] for the definition of $B_{c}$.)

Proof. It suffices to show that if $B$ is a strongly character invariant homogeneous Banach algebra in $S$, then $B \subseteq L^{1}(G) \otimes_{\Gamma} S$. We divide the proof into two steps. First, claim that $B \subseteq L^{l}(G) \otimes \Gamma S$. For any $f \in B$, there exist $\left(g_{n}\right) \subseteq$ $P\left(L^{1}(G)\right)$ and $\left(h_{n}\right) \subseteq B \cap P\left(L^{1}(G)\right)$ such that

$$
f=\sum_{n} g_{n} * h_{n} \text { in }\left(B,\|\|_{B}\right)
$$

and

$$
\sum_{n}\left\|g_{n}\right\|_{1}\left\|h_{n}\right\|_{B}<\infty
$$

which follows immediately from [13, Theorem 3.7(i)] and [11, Theorem 2.6.8]. Since $B \subseteq S$, there exists a constant $\rho$ such that \|\|$_{S} \leqslant \rho\|\|_{B}$, which implies

$$
\begin{aligned}
\sup _{\gamma \in \Gamma} \sum_{n}\left\|\gamma g_{n}\right\|_{1}\left\|\gamma h_{n}\right\|_{S} & \leqslant \rho \sup _{\gamma \in \Gamma} \sum_{n}\left\|g_{n}\right\|_{1}\left\|\gamma h_{n}\right\|_{B} \\
& =\rho \sum_{n}\left\|g_{n}\right\|_{1}\left\|h_{n}\right\|_{B}<\infty,
\end{aligned}
$$

and so $f \in L^{1}(G) \otimes^{\Gamma} S$. Therefore $B \subseteq L^{1}(G) \otimes^{\Gamma} S$. Next, claim that $B \subseteq$ $L^{1}(G) \otimes_{\Gamma} S$. Since $B \subseteq L^{1}(G) \otimes^{\Gamma} S$, it follows that there exists a constant $\rho^{\prime}$ such 
that \|\|$_{\Gamma} \leqslant \rho^{\prime}\|\|_{B}$. For any $f \in B$, we have

$$
\begin{aligned}
\left\|L_{x} f-L_{y} f\right\|_{\Gamma} & \leqslant \rho^{\prime}\left\|L_{x} f-L_{y} f\right\|_{B} \\
& \rightarrow 0 \text { as } y \rightarrow x
\end{aligned}
$$

which implies $f \in L^{l}(G) \otimes_{\Gamma} S$. Therefore $B \subseteq L^{\mathrm{l}}(G) \otimes_{\Gamma} S$. This completes the proof.

\section{A characterization of strongly character invariant Segal algebras}

THEOREM 5. Let $S$ be a Segal algebra on $G$, then the following three properties are equivalent:

(a) There exists a norm under which $S$ becomes a strongly character invariant Segal algebra on $G$.

(b) $L^{1}(G) \otimes_{\Gamma} S=S$.

(c) $\sup \left\{\|\gamma f\|_{S}: \gamma \in \Gamma, f \in P\left(L^{1}(G)\right)\right.$ and $\left.\|f\|_{S}=1\right\}<\infty$.

Proof. Applying Theorem 4 we see that (a) and (b) are equivalent. Now, claim that (b) implies (c). If $L^{1}(G) \otimes_{\Gamma} S=S$, then there exists a constant $\rho$ such that $\|f\|_{S} \leqslant\|f\|_{\Gamma} \leqslant \rho\|f\|_{S}$ for all $f \in S$. This implies that for any $f \in P\left(L^{\prime}(G)\right)$ we have

$$
\sup _{\gamma \in \Gamma}\|\gamma f\|_{S} \leqslant \sup _{\gamma \in \Gamma}\|\gamma f\|_{\Gamma}=\|f\|_{\Gamma} \leqslant \rho\|f\|_{S}
$$

It follows that

$$
\sup \left\{\|\gamma f\|_{S}: \gamma \in \Gamma, f \in P\left(L^{1}(G)\right) \text { and }\|f\|_{S}=1\right\} \leqslant \rho<\infty,
$$

which shows that (b) implies (c). Next, claim that (c) implies (b). Assume that

$$
\rho=\sup \left\{\|\gamma f\|_{S}: \gamma \in \Gamma, f \in P\left(L^{1}(G)\right) \text { and }\|f\|_{S}=1\right\}<\infty .
$$

In view of Proposition 3, it suffices to show that $S \subseteq L^{\prime}(G) \otimes_{\Gamma} S$. The proof of Theorem 4 can be applied to this case if we use this $\rho$ to play its role in Theorem 4 and replace $(*)$ in Theorem 4 by

$$
\begin{aligned}
\sup _{\gamma \in \Gamma} \sum_{n}\left\|\gamma g_{n}\right\|_{1}\left\|\gamma h_{n}\right\|_{S} & =\sup _{\gamma \in \Gamma} \sum_{n}\left\|g_{n}\right\|_{1}\left\|\gamma h_{n}\right\|_{S} \\
& \leqslant \rho \sum_{n}\left\|g_{n}\right\|_{l}\left\|h_{n}\right\|_{S}<\infty .
\end{aligned}
$$

It is so easy as to be omitted.

ExAmPLE. Consider the following Segal algebras:

(a) $C^{k}(T)$ consists of all $k$-times continuously differentiable functions on $T$, with the norm $\|f\|=\sup _{0 \leqslant j \leqslant k}\left\|f^{(j)}\right\|_{\infty}$. 
(b) $L^{(k)}(T)$ consists of all $f$ in $L^{1}(T)$ such that for $j=0,1, \ldots, k-1, f^{(j)}$ are absolutely continuous on $T$ and $f^{(j+1)} \in L^{\prime}(T)$, with the norm $\|f\|=$ $\sup _{0 \leqslant j \leqslant k}\left\|f^{(j)}\right\|_{1}$.

(c) $L^{(k)}(R)$ consists of all $f$ in $L^{1}(R)$ such that for $j=0,1, \ldots, k-1, f^{(j)}$ are absolutely continuous on $R$ and $f^{(j+1)} \in L^{1}(R)$, with the norm $\|f\|=$ $\sup _{0 \leqslant j \leqslant k}\left\|f^{(j)}\right\|_{1}$ (see [1], [6], [8], [12], [13]). Let $S$ denote any one of $C^{k}(T)$, $L^{(k)}(T)$ and $L^{(k)}(R)$. It is well-known that $S$ is character invariant. From Theorem 5 it is easy to show that there exists no norm under which $S$ becomes a strongly character invariant Segal algebra.

\section{A characterization of ideals in $L^{1}(G)$ which contain a strongly character invariant Segal algebra}

THEOREM 6. Let $S$ be a Banach ideal in $L^{1}(G)$; then the following three properties are equivalent:

(a) There is the largest among all strongly character invariant Segal algebras in $S$.

(b) $S$ contains a strongly character invariant Segal algebra on $G$.

(c) $P\left(L^{\prime}(G)\right) \subseteq S$ and $\sup _{\gamma \in \Gamma}\|\gamma f\|_{S}<\infty$ for all $f \in P\left(L^{\prime}(G)\right)$.

Proof. Applying Theorem 4 we see that (a) and (b) are equivalent. Now, claim that (b) implies (c). Let $B$ be a strongly character invariant Segal algebra in $S$. Then $P\left(L^{\mathrm{l}}(G)\right) \subseteq S$ and there exists a constant $\rho$ such that \|\|$_{S} \leqslant \rho\|\|_{B}$. This implies that for any $f \in P\left(L^{\mathrm{l}}(G)\right)$ we have

$$
\sup _{\gamma \in \Gamma}\|\gamma f\|_{S} \leqslant \rho \sup _{\gamma \in \Gamma}\|\gamma f\|_{B}=\rho\|f\|_{B}<\infty .
$$

This shows that (b) implies (c). Next, claim that (c) implies (a). In view of Theorem 4, it suffices to show that $P\left(L^{1}(G)\right) \subseteq L^{1}(G) \otimes_{\Gamma} S$. For any $f \in P\left(L^{1}(G)\right)$ there exists $g \in P\left(L^{\prime}(G)\right)$ such that $\hat{g}=1$ on supp $\hat{f}$. This implies that

$$
\begin{aligned}
f & =g * f, \\
\sup _{\gamma \in \Gamma}\|\gamma g\|_{1}\|\gamma f\|_{S} & =\|g\|_{1} \sup _{\gamma \in \Gamma}\|\gamma f\|_{S}<\infty
\end{aligned}
$$

and

$$
\begin{aligned}
\left\|L_{x} f-L_{y} f\right\|_{\Gamma} & =\left\|\left(L_{x} g-L_{y} g\right) * f\right\|_{\Gamma} \leqslant \sup _{\gamma \in \Gamma}\left\|\gamma\left(L_{x} g-L_{y} g\right)\right\|_{1}\|\gamma f\|_{S} \\
& =\left\|L_{x} g-L_{y} g\right\|_{1} \sup _{\gamma \in \Gamma}\|\gamma f\|_{S} \\
& \rightarrow 0 \text { as } y \rightarrow x .
\end{aligned}
$$


It follows that $f \in L^{\prime}(G) \otimes_{\Gamma} S$ and so $P\left(L^{\prime}(G)\right) \subseteq L^{\prime}(G) \otimes_{\Gamma} S$. This completes the proof.

EXAMPLE. Let $\alpha$ be a locally bounded function on $\Gamma$ with $\alpha \geqslant 1$. Define $S(\alpha)$ as the space of all $f$ in $L^{1}(G)$ such that $\lim \hat{f}(\gamma) \alpha(\gamma)=0$. Under the norm $\|f\|_{\alpha}=$ $\|f\|_{1}+\sup _{\gamma \in \Gamma}|\hat{f}(\gamma) \alpha(\gamma)|, S(\alpha)$ forms a Segal algebra on $G$. (See [10].) We claim that $S(\alpha)$ contains no strongly character invariant Segal algebras on $G$ if and only if $\alpha$ is unbounded on $\Gamma$. In this case, it follows that $L^{\prime}(G) \otimes_{\Gamma} S(\alpha)$ is not a Segal algebra on $G$. Now we give a detailed proof as follows: Take $f \in P\left(L^{\prime}(G)\right)$ with $\hat{f}(0)=1$. We have

$$
\begin{aligned}
\sup _{x \in \Gamma}|\alpha(\chi)| & =\sup _{\chi \in \Gamma}|\hat{f}(0) \alpha(\chi)| \\
& \leqslant \sup _{\chi \in \Gamma} \sup _{\gamma \in \Gamma}|\hat{f}(\gamma-\chi) \alpha(\gamma)| \\
& =\sup _{\chi \in \Gamma} \sup _{\gamma \in \Gamma}\left|(\chi f)^{\wedge}(\gamma) \alpha(\gamma)\right| \leqslant \sup _{\chi \in \Gamma}\|\chi f\|_{\alpha} .
\end{aligned}
$$

If $\alpha$ is unbounded on $\Gamma$, then $\sup _{\chi \in \Gamma}\|\chi f\|_{\alpha}=\infty$. From Theorem 6 we find that $S(\alpha)$ contains no strongly character invariant Segal algebras on $G$. If $\alpha$ is bounded on $\Gamma$, [10, Proposition 2] states that $S(\alpha)=L^{1}(G)$, which is a strongly character invariant Segal algebra on $G$. This completes the proof.

ExAmple. Define $F(R)$ as the space of all $f$ in $L^{\mathrm{l}}(R)$ such that $\lim \hat{f}(n) \log n=0$. Under the norm $\|f\|=\|f\|_{1}+\sup _{n}|\hat{f}(n)| \log n, F(R)$ forms a Segal algebra on $R$ (see [4]). In this case, $G=R, \Gamma=R$ and so the largest strongly character invariant homogeneous Banach algebra in $F(R)$ is $L^{\prime}(R) \otimes_{R} F(R)$. By a similar argument as above we can show that $F(R)$ contains no strongly character invariant Segal algebras on $R$ and $L^{1}(R) \otimes_{R} F(R)$ is not a Segal algebra on $R$.

\section{Acknowledgement}

I would like to thank the referee for his valuable suggestions.

\section{References}

[1] C. P. Chen, 'On the intersections and the unions of Banach algebras,' Tamkang J. Math. 9 (1978), 21-27.

[2] J. Cigler, 'Normed ideals in $L^{\prime}(G)$,' Neder!. Akad. Wetensch, Indag. Math. 31 (1969), 273-282.

[3] H. G. Feichtinger, 'Multipliers from $L^{\prime}(G)$ to a homogeneous Banach space.' J. Math. Anal. Appl. 61 (1977), 341-356. 
[4] R. R. Goldberg, Recent results on Segal algebras (Springer-Verlag, Lecture Notes in Math. 399. 1974).

[5] E. Hewitt and K. A. Ross, Abstract harmonic analysis (Springer-Verlag, New York, I (1963), II (1970)).

[6] Y. Katznelson, An introduction to harmonic analysis (Wiley, New York, 1968).

[7] V. Losert, 'A characterization of the minimal strongly character invariant Segal algebra,' Ann. Inst. Fourier 30 (1980), 129-139.

[8] H. Reiter, Classical harmonic analysis and locally compact groups (Oxford Mathematical Monographs, Oxford University Press, 1968).

[9] H. Reiter, $L^{1}$-algebras and Segal algebras (Lecture Notes in Math. No. 231, Springer-Verlag, Berlin, 1971).

[10] M. Riemersma, 'On some properties of normed ideals in $L^{1}(G)$,' Nederl. Akad. Wetensch. Proc. Ser. $A 78$ = Indag. Math. 37 (1975), 265-272.

[11] W. Rudin, Fourier analysis on groups (Interscience, New York, 1962).

[12] H. C. Wang, 'Nonfactorization in group algebras,' Studia Math. 42 (1972), 231-24].

[13] H. C. Wang, Homogeneous Banach algebras (Lecture Notes in Pure and Appl. Mathematics, Dekker, New York, 1977).

Institute of Mathematics

National Tsing Hua University

Hsinchu, Taiwan 300

Republic of China
Author's current address

Department of Mathematics

Stanford University

Stanford, California 94305

U.S.A. 